\title{
Haemophilic arthropathy: a case of misdiagnosis
}

Nakalyango Annette Grace

This article describes a case of wrongly managed haemophilic arthropathy in a 15-year-old boy in Uganda. Delayed diagnosis of haemophilia and misdiagnosis of haemophilic arthropathy resulted in unnecessary corrective surgery and potentially severe complications. After haemophilia was diagnosed, he was managed by haematologists using factor VIII and regular physiotherapy. The physiotherapy treatment approach focused in particular on functional activities, which was beneficial in achieving quicker results and made the patient's rehabilitation more meaningful. This case indicates that there is a need for greater awareness of the presentation of haemophilia in developing countries.

Keywords: Arthrofibrosis, arthropathy, haemarthrosis, misdiagnosis, physiotherapy, Uganda

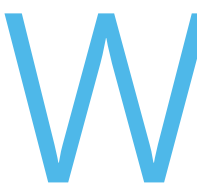

ith a population of around 45 million people in Uganda, there should be approximately 2,250 people diagnosed with haemophilia. However, services for people with haemophilia in Uganda are poor, resulting in very low number of diagnoses and limited access to haemophilia care. Since joining a World Federation of Hemophilia twinning programme supporting diagnosis programmes, there are now 213 confirmed cases of haemophilia across. The majority of diagnosis and

NAKALYANGO ANNETTE GRACE

Physiotherapist, Mulago National Referral Hospital,

Kampala, Uganda

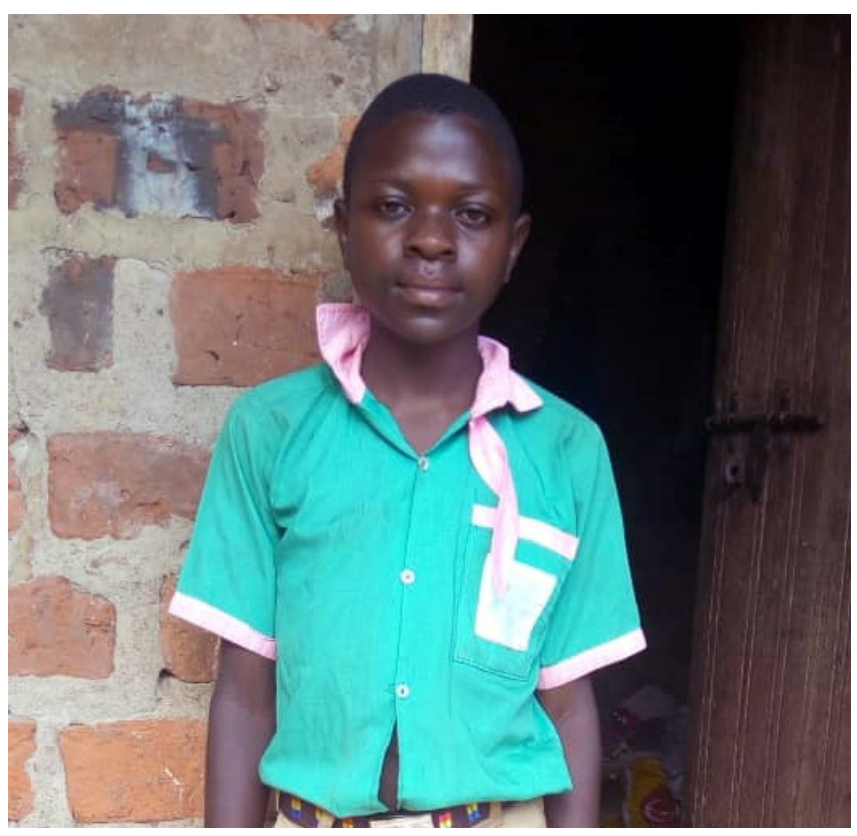

The case of a young patient who was misdiagnosed with and treated for septic arthritis, instead of haemophilic arthropathy, raises issues around the need for greater awareness of bleeding disorders in developing countries such as Uganda.

treatment occurs via Mulago Hospital in Kampala, the national haemophilia referral centre. Due to limited supply of coagulation factors, most of which are donated via humanitarian aid, there is virtually no prophylaxis. A very small number of patients have factor at home which is administered on-demand via regional hospitals to minimise travel to Kampala and to speed up infusion time post-bleed. Many relatively young people with haemophilia have evidence of early haemophilia-related joint disease from recurrent, untreated haemarthrosis.

Haemarthrosis leads to changes within the joints, including synovial proliferation, which results in

This is an Open Access article distributed under the terms of the Creative Commons Attribution-NonCommercial-NoDerivs License (https://creativecommons.org/licenses/by-nc-nd/3.0/) which permits use and distribution in any medium, provided the original work is properly cited, the use is non-commercial, and no modifications or adaptations are made. Copyright is retained by the authors. 


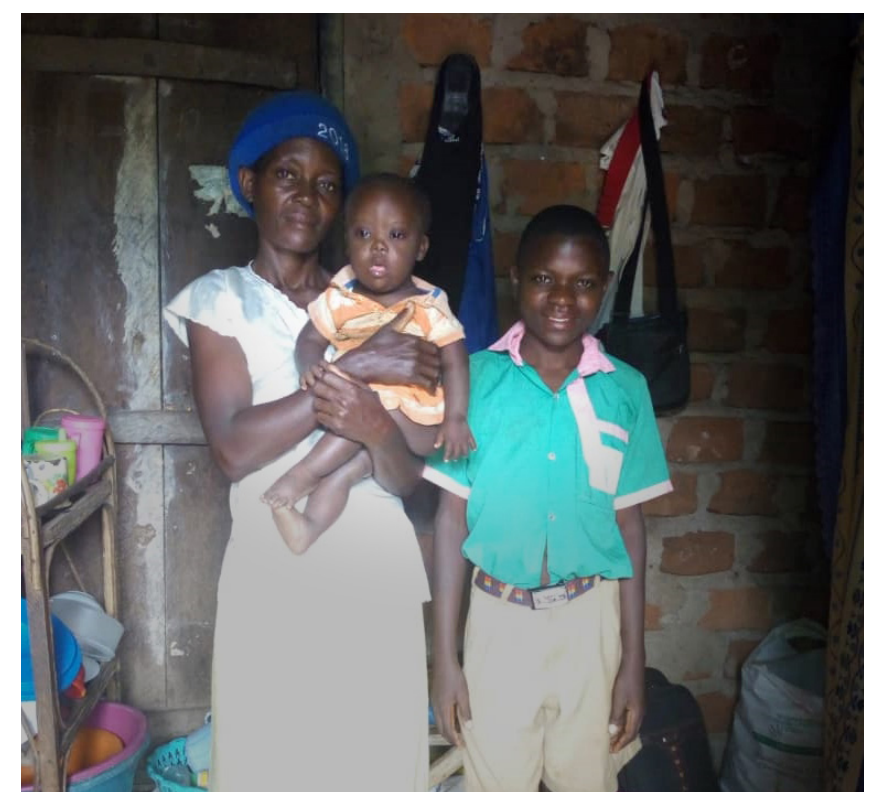

The patient lives with his mother, his paternal grandmother and eight brothers and sisters in a one-room house. One brother has also been diagnosed with haemophilia and two sisters show bleeding tendencies; another brother died from bleeding most probably related to undiagnosed haemophilia

further bleeding and chronic synovitis, and progressive destruction of both cartilage and bone. The end result is known as haemophilic arthropathy, which is characterised by pain, stiffness and deformity. The joint most commonly affected is the knee. Haemophilic arthropathy is rarely considered a differential diagnosis of knee swelling and many patients undergo orthopaedic intervention before a possible bleeding disorder is considered. We report such a case here, with a now 15-year-old young man.

\section{PATIENT HISTORY}

The patient comes from a large family who live in constrained socioeconomic conditions in a rural area approximately three hours by road from Kampala. The patient's mother is separated from the father of her children and has no formal employment. The family live in a small one-room house which is poorly furnished, together with their paternal grandmother. There are nine children: four boys and five girls. Two of the boys have now been diagnosed with haemophilia: the patient, and a younger brother who also has Down's syndrome. Two sisters have frequent nosebleeds; the other children do not show any bleeding tendencies. There are also two maternal uncles who were diagnosed with haemophilia after the patient. One other brother and two maternal uncles died from bleeding presumed to be due to undiagnosed haemophilia.

The patient and four of his siblings attend school; today the patient has a bursary for his school fees through the charity Save One Life, but his siblings are occasionally sent home due to non-payment of school fees. He rides a bicycle to school, which is three kilometres from home along a bumpy, narrow road with no tarmac.

As a child, the patient experienced bleeding all over his arms and skin, from injection sites and after tooth extraction, and occasionally had gum bleeds. At five years of age he had a major swelling in the right knee, which became his target joint - in hindsight, this swelling was a bleed. Despite occasional bruises while growing up, the patient attained all his developmental milestones at the right time, although he had a valgus deformity of the right knee and fixed knee extension.

Recurrent bleeds over many years left the patient with an extremely swollen right knee, with limited movement and function. This prompted the boy's mother to seek help from ACHERU, a charity that assesses and treats children with disabilities, in 2014, when he was 11 years old. The charity referred him to CoRSU Rehabilitation Hospital in Kampala, which specialises in providing orthopaedic and reconstructive surgical interventions and rehabilitation services for children with physical impairments.

At CoRSU, the patient was diagnosed with septic arthritis of the right knee and was booked in for corrective surgery. An arthrotomy and biopsy were performed on 12 May 2014. After surgery, a full leg plaster of Paris cast was applied, which was soaked in blood shortly after. This was replaced with a back slab and the patient was discharged from hospital to home. After one week, the stitches were removed, but the wound gave way and bleeding increased. The patient was readmitted to CoRSU, where he was taken back to theatre for stitching. He was then referred to Mulago National Referral Hospital in Kampala, where he was diagnosed with moderate haemophilia $A$ and infused with factor VIII. The bleeding ceased, and two days later, he was referred for physiotherapy.

On presentation to the physiotherapy department the patient had an extremely painful, swollen right knee, which was maintained in extension. He was in obvious pain despite analgesia, including morphine, and unable to move any of the joints of his right lower limb. He was unable to even get the toes of his right foot to the floor, making weight-bearing mobilisation impossible. He was provided with crutches to assist mobility, but due to the instability of the terrain (ruts in mud roads, mud becoming sticky due to rainfall, etc.) his mobility was limited to within his home, meaning that he was unable to attend school, church or local gatherings. 

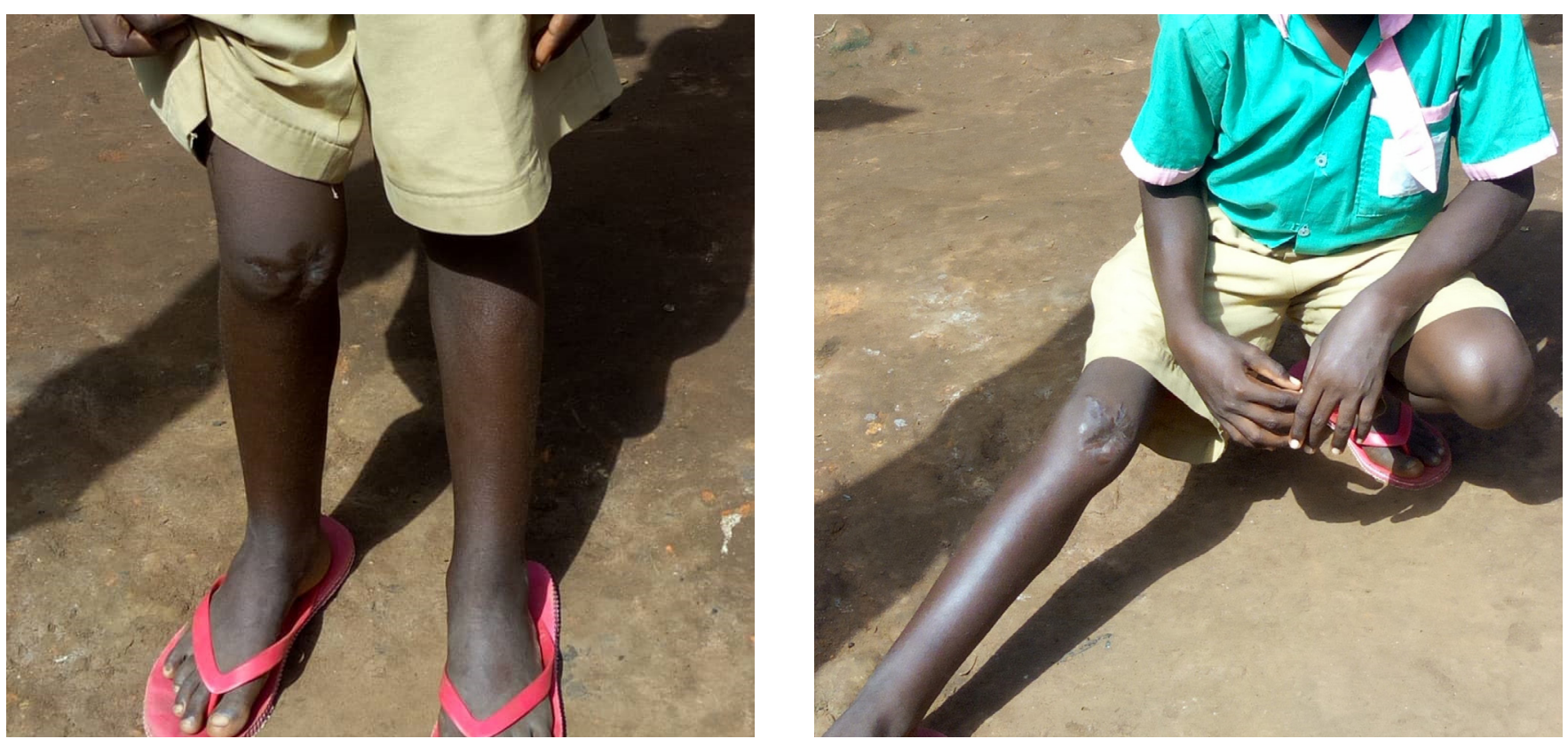

When the patient presented to the physiotherapy department at Mulago Hospital, he had a valgus deformity on the right knee, which was fixed in extension. Through a programme of physiotherapy focusing on functional activities, the condition of his knee and general quality of life have improved significantly

\section{Examination of the upper limbs}

There were no bruises noted on the skin. There was a small swelling on the right fore arm; however, range of motion of the right elbow was normal and function preserved. The patient had full range of motion in the right shoulder, despite bleeding in the shoulder a week before. The left arm had normal movements and function.

\section{Examination of the lower limbs}

There were no bruises seen on the lower limbs. The right knee had a valgus deformity. The knee was fixed in extension, with flexion limited to less than 10 degrees. The range of movement of the left knee was normal. Both ankle joints were normal.

\section{Function abilities}

Before this acute episode the patient could stand and walk without support, stand on one leg and squat on the left leg. This allowed him to use the latrine. He could previously ride a bicycle. However, he had an adapted gait, with the knee in internal rotation.

\section{Difficulties}

The patient had difficulty squatting on both legs and kneeling on four points.

\section{DIAGNOSIS AND MANAGEMENT}

The patient was diagnosed with arthrofibrosis of the right knee and haemophilia arthropathy.
Arthrofibrosis of the knee is a condition whereby knee motion is limited due to scar tissue, contractures after injury or surgery, or due to the effects of osteoarthritis over time. This diagnosis was based on the presence of stiffness, the patient's inability to straighten or flex the knee, and his inability to perform a full revolution on his bicycle, which he had been using for mobility within his rural environment.

Several potential problems were identified:

- The risk of bleeding from a fall as a result of cycling $n$ a bumpy road;

- The risk of upper limb bleeds due to use of crutches causing additional weight bearing stress on wrists/ elbows/shoulders:

- Difficulty accessing regular follow-ups due to socioeconomic constraints.

A four-point plan of management was created for the patient. This involved:

1. Strengthening all muscles in the patient's legs, including the hip and ankle;

2. Doing a mixture of exercises, both with and without resistance, including hydrotherapy and Therabands;

3. Using functional activities, such as gentle mobilisation and one leg standing, on his right leg, to work on muscle and functional skills, with the aim of regaining functional ability to enable the usual activities of daily living;

4. Practicing to achieve as close to normal gait as possible. 
As a result of the patient's diagnosis with haemophilia A and referral for treatment with a physiotherapist, the condition of his right knee and his quality of life in general has improved. He is now able to walk without support and can ride a bicycle correctly. He attends school regularly and will be sitting his primary leaving examinations in 2019. At the time of writing, he has not experienced a bleed in his right knee since 2015 .

\section{CONCLUSION}

This case is a good example of how wrong diagnosis leads to wrong management, which may then result in disability or worse outcomes for the patient. There is still a great need for more awareness of haemophilia and the associated problems that can arise as a result of bleeding disorders among medical practitioners in Uganda and other developing countries. In terms of physiotherapy for this patient, focusing on functional activities gave quicker results and made his rehabilitation more meaningful. This is therefore recommended as a beneficial approach in physiotherapy treatment for arthrofibrosis and haemophilic arthropathy. The role of physiotherapyled rehabilitation should not be underestimated - the impact for this young man and his family has been enormous; enabling him to return to his previous functional ability and family way of life.

\section{ACKNOWLEDGEMENTS}

The authors have advised no interests that might be perceived as posing a conflict or bias.

Informed consent has been obtained from the individual reported in this case study.

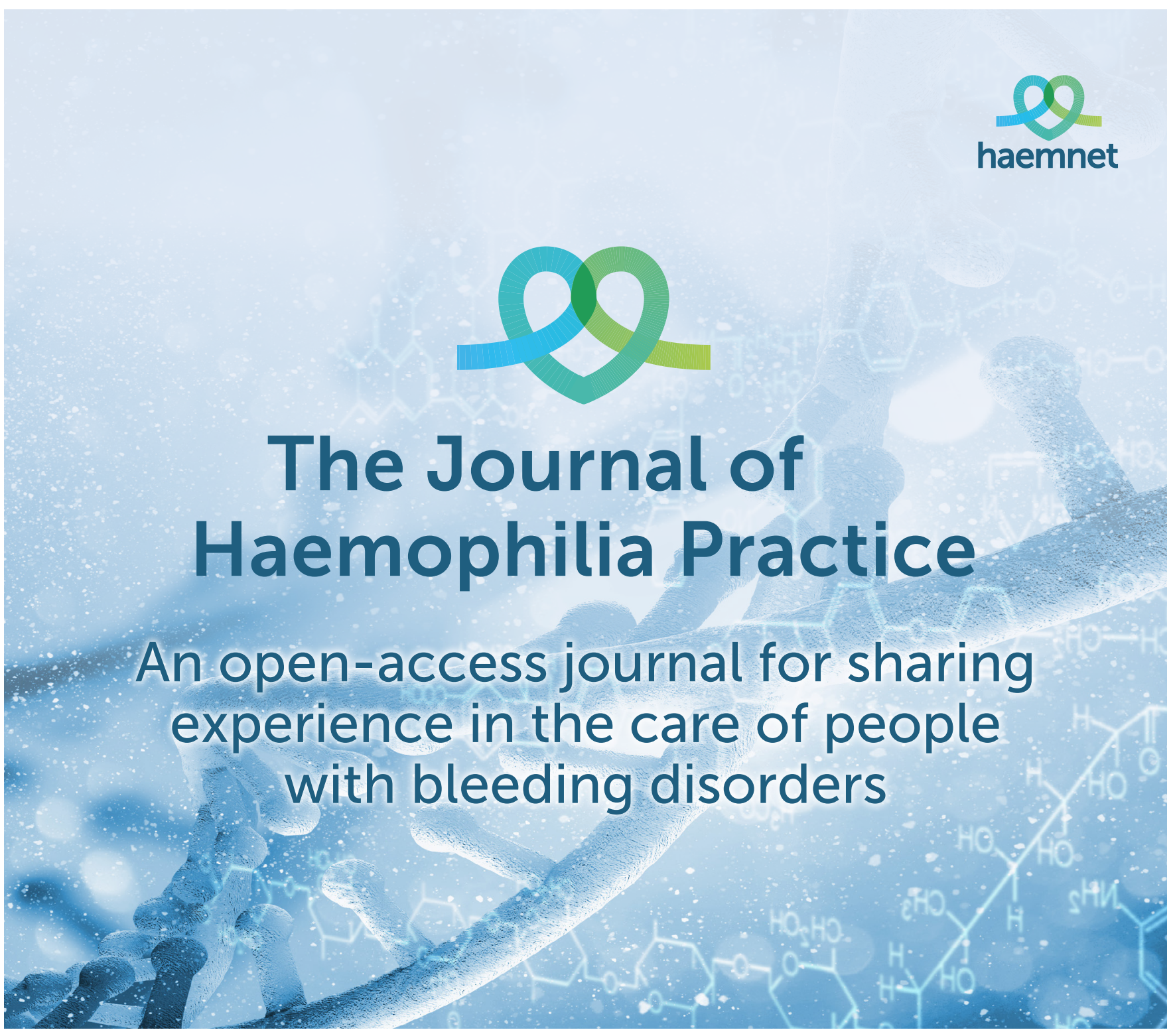

